\title{
The carrier-multipath observable: a new carrier- phase multipath mitigation technique
}

\author{
Ramin Moradi, Wolfgang Schuster, Shaojun Feng, Altti Jokinen, Washington Ochieng
}

Imperial College London, South Kensington Campus, London SW7 2AZ, UK

e-mail:rm4310@imperial.ac.uk; R.Moradi10@imperial.ac.uk

\begin{abstract}
Modeling and mitigating carrier-phase multi-path errors continue to be a significant challenge for high-accuracy positioning using global navigation satellite sys-tems. The multipath error is dependent on the operational environment and therefore cannot be mitigated by differ-encing techniques. The effect of multipath is accentuated when observables based on linear combinations of mea-surements from two or more frequencies are formulated. We develop a new carrier-phase multipath error observable that isolates the inter-frequency carrier-phase multipath error for linear combinations of observables, such as wide-lane (WL). Realtime kinematic positioning results from varying baseline lengths show that a significant reduction in between 14 and $47 \%$ in the time to initial ambiguity resolution are achieved by correcting the WL observable using the new carrierphase multipath error observable.
\end{abstract}

Keywords Linear combination · Inter-frequency multipath $\cdot$ Wide lane $\cdot$ Carrier-phase multipath

\section{Introduction}

Positioning with global navigation satellite systems (GNSS) uses two types of range measurements: code phase and carrier phase. For applications requiring high-posi-tioning accuracy, carrier-phase measurements are typically used. However, various error sources prevent high-accu-racy positioning, including a lack of synchronization between satellite and receiver clocks and atmospheric errors. Satellite and receiver clock errors can be eliminated by differencing techniques. Atmospheric errors can also be significantly mitigated for short baselines with differencing techniques. Additionally, measurements are affected by multipath, which occurs when a signal reflected from surrounding objects reaches the antenna in addition to the lineof-sight (LOS) signal. This type of error is highly variable, typically uncorrelated between-receivers even with very short baselines and is therefore difficult to mitigate by differencing (Lau and Cross 2007b).

For short baseline conventional RTK positioning, e.g., less than $10 \mathrm{~km}$, after mitigating the correlated errors through differencing, the range is obtained by combining the fractional carrier-phase measurement with the number of whole carrier cycles in the propagation path. The latter are computed using both code and carrier measurements in a process known as integer-ambiguity resolution. This typically consists of two steps: a least-squares method to derive the float solution followed by a search in the integer domain. Reliable resolution is a key factor in high-accuracy relative GNSS positioning (Jokinen et al. 2012). This is a challenging task and computationally demanding. One way to facilitate this procedure is to increase the effective wavelength, by combining measurements at two different frequencies. However, this increases noise and multipath errors, potentially leading to biased estimations 
of the float solutions and thereby impacting ambiguity resolution.

Existing multipath detection and mitigation techniques can be classified into antenna, signal and measurement processing based techniques. Antenna-based techniques exploit the sensitivity to signal polarization. However, mitigation performance depends on the signal elevation angle and antenna gain pattern (Manandhar et al. 2004), resulting for example, in poor mitigation performance for low elevation reflections (Brenneman et al. 2007). Signal processing techniques mitigate multipath mainly by adjusting correlator spacing. However, these can only mitigate long-delay multipath and are not suitable to mit-igate short-delay multipath (Van Dierendonck et al. 1992). Therefore, after applying both antenna and signal pro-cessing techniques, measurement processing techniques are typically used to mitigate residual multipath errors. These include signal to noise ratio (SNR), antenna array and ray-tracing techniques, as well as stochastic models. The common limitation of the first three methods is that the number of reflected signals must be known. This is gen-erally not realistic, especially in varying operational envi-ronments. In addition, SNR-based techniques suffer from an unknown time lag between SNR and multipath. Antenna arrays also have limited applications due to their relatively large size. In ray tracing, the satellite-reflectorantenna geometry must be known. Due to these limitations, sim-plified stochastic models are typically used to reduce the impact of multipath in the measurement domain. For example, in the elevation-based stochastic model, all low elevation signals are de facto down-weighted because they are most prone to multipath. In the SNR-based stochastic model, the correlation between SNR and multipath is used to weight the measurements. However, the time lag between SNR and multipath is a limiting factor in this model (Lau and Cross 2007a, b).

The limitations above are addressed by developing a new model to detect and mitigate carrier-phase multipath errors of linear combinations with the aim to reduce ambiguity resolution times for the wide-lane (WL) obser-vable (Joosten and Irsigler 2003). The observation models are introduced first and then carrier-multipath error models for the singlefrequency case as well as for linear combi-nations are developed. The concept of the inter-frequency carriermultipath (IFM) observable first introduced in (Moradi et al. 2013) is expanded for single-differencing between-satellites, single-differencing between-receivers and double-differencing modes. The methodology to mit-igate carrier-multipath error using the IFM observable for observables obtained from linear combinations is then developed and evaluated with real data.

\section{Carrier-phase multipath error model}

The carrier-phase measurement can be expressed as follows:

$$
\begin{aligned}
\Phi_{f, i}^{k}= & \rho_{i}^{k}-I_{f, i}^{k}+T_{i}^{k}-\lambda_{f} N_{f, i}^{k}+\lambda_{f} m_{f, i}^{k}+c t_{\mathrm{r}, i}+c t_{\mathrm{s}, i}+b_{f}^{k} \\
& +B_{f, i}+\varepsilon
\end{aligned}
$$

where $k$ is the satellite index, $f$ the frequency, $i$ the receiver index, $\Phi$ the carrier-phase measurement, $\rho$ the geometric range, $I$ the ionospheric-induced error, $T$ the tropospheric delay, $\lambda$ the carrier-phase wavelength, $N$ the integer ambiguity in cycles, $m$ the carrier-phase multipath error in cycles, $c$ the speed of light, $t_{\mathrm{r}}$ the receiver clock bias, $t_{\mathrm{s}}$ the satellite clock bias, $b$ the satellite hardware delay, $B$ the receiver hardware delay and $\varepsilon$ the measurement noise.

Multipath errors occur when signals from a satellite are reflected off the surface of an object in the vicinity of the antenna and received together with the direct signals. The two-dimensional multipath geometry with a vertical reflector is shown in Fig. 1.

Using the geometry relation, the phase shift due to multipath in radians is obtained as:

$\gamma=\frac{2 \pi}{\lambda} d \cos (e)$

where $e$ is the elevation angle, $d$ is distance between the antenna and its image and $\gamma$ is the phase shift. Using the phase shift $\gamma$ and reflection coefficient $\Gamma$, the carrier-phase multipath error is obtained as (Reichert and Axelrad 1999):

$m=\tan ^{-1}\left(\frac{\Gamma \sin \gamma}{1+\Gamma \cos \gamma}\right)$

From (3), the theoretical maximum of the carrier-multipath error is $0.25 \lambda$ for a maximum reflection coefficient of 1 . This corresponds to 0.046 and $0.061 \mathrm{~m}$ for GPS L1 and L2 carrier-phase measurements, respectively. However, reflective surfaces with the maximum reflection

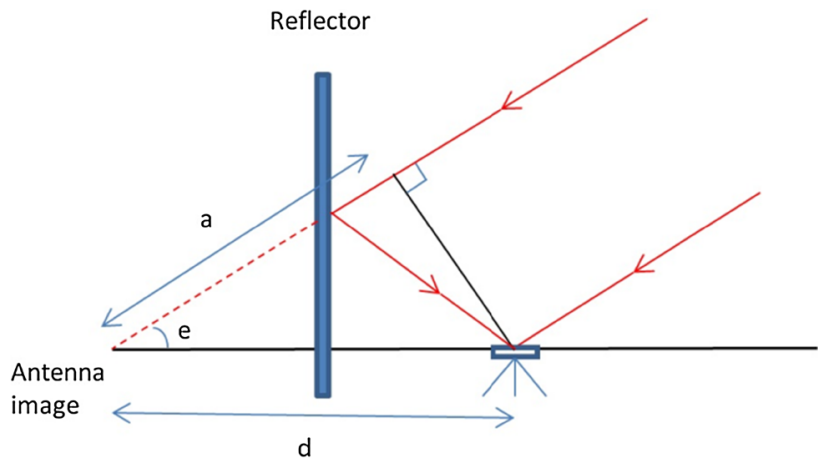

Fig. 1 Multipath geometry 
coefficient are rare in practice. Typically, errors are smaller than the maximum theoretical values and can be ignored for most applications. However, observables obtained from linear combinations of other observables have significantly higher carrier-multipath errors and cannot be ignored.

\section{Carrier-phase multipath error in linear combinations}

A linear combination observable such as WL can be generalized by the following equation (Wanninger and May 2001):

$V_{c}=\alpha V_{1}-\beta V_{2}$

where $V_{1}$ and $V_{2}$ are the observable or multipath errors of the original signals in units of length, $V_{c}$ is the observable or multipath error of the linear combination in units of length and $\alpha$ and $\beta$ are coefficients that define the relation between the observable or multipath errors in the combination. According to (2), (3) and (4) the maximum carrier-multipath error $m_{c, \max }$ in a linear combination are:

$m_{c, \max }=\alpha m_{1, \max }+\beta m_{2, \max }$

In the WL observable based on GPS L1 and L2 observables, $\alpha$ and $\beta$ are obtained as follows:

$\alpha_{\mathrm{wl}}=\frac{f_{1}}{f_{1}-f_{2}} \approx 4.53$

$\beta_{\mathrm{wl}}=\frac{f_{2}}{f_{1}-f_{2}} \approx 3.53$

This leads to maximum values of the WL carrier-multipath error $m_{w i \text { max }}$ equal to $0.43 \mathrm{~m}$. This is significantly higher than the error at each individual frequency. Relationships between the simulated multipath errors in L1, L2 and the WL observables are shown in Fig. 2. The multipath errors were generated as a function of multipath delay $d$ using (2) and (3) with an elevation angle of $15^{\circ}$ and a reflection coefficient of 0.5 . The significant increase in multipath error of the WL in comparison to the L1 and L2 carrier-phase measurements can be seen. The figure shows that the WL multipath error increases as a result of increasing multipath phase differences between the L1 and L2 carriers. This increase makes ambiguity resolution more difficult.

\section{IFM observable}

The IFM observable is derived from the combined use of measurements at different frequencies such as GPS L1 and L2. Depending on the application, the IFM observable can be obtained in different modes, namely between-receivers,

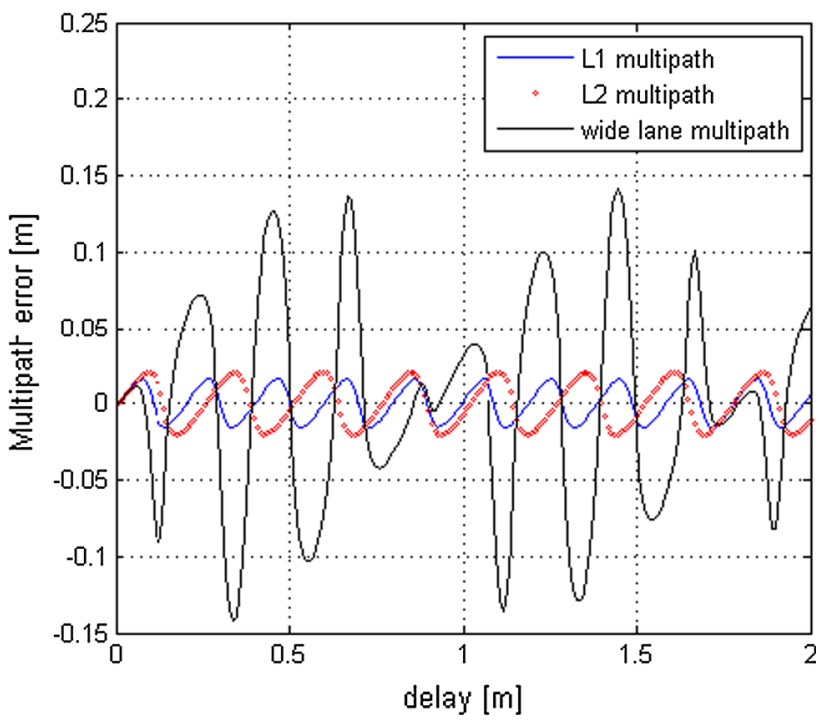

Fig. 2 Relation between simulated multipath errors in L1, L2 and WL for reflection coefficient of 0.5

between-satellites and double-differenced modes. These modes are explained in the next three subsections.

Between-receivers mode

The IFM observable in this mode is obtained in three steps. In the first step, the between-receiver single-difference of the carrier-phase measurements is formed for each frequency:

$$
\begin{aligned}
\Delta \Phi_{f}^{k}= & \Delta \rho^{k}-\Delta I_{f}^{k}+\Delta T^{k}-\lambda_{f} \Delta N_{f}^{k}+\Delta m_{f}^{k}+c \Delta t_{r} \\
& +\Delta B_{f}+\varepsilon^{\prime}
\end{aligned}
$$

where $\Delta$ represents the differencing between-receivers and $\varepsilon^{\prime}$ represents noise in the single-differenced measurements. This operation eliminates common errors between the two receivers and, for short baselines, decreases atmospheric errors significantly due to the error correlation.

Second, single-difference measurements are then differenced across frequencies to eliminate receiver clock errors and the geometric ranges:

$$
\begin{aligned}
\Delta \Phi_{\mathrm{L} 1}^{k}-\Delta \Phi_{\mathrm{L} 2}^{k}= & -\Delta I_{\mathrm{L} 1}^{k}+\Delta I_{\mathrm{L} 2}^{k}-\lambda_{\mathrm{L} 1} \Delta N_{\mathrm{L} 1}^{k}+\lambda_{\mathrm{L} 2} \Delta N_{\mathrm{L} 2}^{k} \\
& +\Delta m_{\mathrm{L} 1}^{k}-\Delta m_{\mathrm{L} 2}^{k}+\Delta B_{\mathrm{L} 1}-\Delta B_{\mathrm{L} 2}+\varepsilon^{\prime \prime}
\end{aligned}
$$

where $\varepsilon^{\prime \prime}$ represents the measurement noise after differencing across frequencies. These two steps significantly reduce the residual ionospheric error for short- and medium-baselines, as well as the rate of change of the residual ionospheric error. The residual ionospheric error in (9) is the inter-frequency ionospheric error $\Delta I_{\mathrm{L} 2, \mathrm{~L} 1}$. This error can be expressed as a function of 
the L2 ionospheric error, using the relationship between L1 ionospheric error $I_{\mathrm{L} 1}$ and L2 ionospheric errors $I_{\mathrm{L} 2}$ :

$$
\begin{aligned}
& I_{\mathrm{L} 1}=\frac{f_{2}^{2}}{f_{1}^{2}} I_{\mathrm{L} 2} \\
& \Delta I_{\mathrm{L} 2, \mathrm{~L} 1}=I_{\mathrm{L} 2}-\frac{f_{2}^{2}}{f_{1}^{2}} I_{\mathrm{L} 2}=0.39 I_{\mathrm{L} 2}
\end{aligned}
$$

The third step is designed to remove the residual ionospheric error, the ambiguity terms and the differential hardware delays $\left(\Delta B_{\mathrm{L} 1}-\Delta B_{\mathrm{L} 2}\right)$ between the L1 and L2 frequencies. Ambiguity terms are constant provided that cycle slips are detected and repaired. The differential hardware delays between the L1 and L2 frequencies, also known as inter-frequency biases (IFB), are stable over long durations of the order of days, and can therefore, be treated as constant (Gao et al. 1994). Hence, the only remaining variable term in addition to the multipath errors after step two is residual ionospheric error. It is known that differenced ionospheric error increases approximately $2 \mathrm{~mm} / \mathrm{km}$ baseline under nominal ionospheric conditions (Christie et al. 1998). This can be assured with appro-priate ionospheric monitoring (Schuster and Ochieng 2011). Under these conditions, based on (11), we can approximate the residual ionospheric error increase as $0.78 \mathrm{~mm} / \mathrm{km}$ baseline. If the ionospheric conditions are constant, the changes in the residual ionospheric errors resulting from changes in the elevation angle over time can be extracted using the single-layer mapping (SLM) function (Komjathy and Langley 1996; Mannucci et al. 1998). In this function it is assumed that all the free electrons are concentrated in an infinitesimal spherical layer around the earth. The SLM is used to calculate the relation between vertical TEC and slant TEC values based on:

$$
\mathrm{SLM}=\frac{1}{\cos \left(z^{\prime}\right)}
$$

where $z^{\prime}$ is the geocentric zenith distance at the ionospheric pierce point. The angle $z^{\prime}$ is obtained from the geocentric zenith distance $z$ at the height of the receiver based on:

$z^{\prime}=\arcsin \left(\frac{R}{R+H} \sin (z)\right)$

where $R$ is approximately $6,371 \mathrm{~km}$, the mean radius of the earth and $H$ is the height of the assumed single layer, which is usually set to $350-450 \mathrm{~km}$. A value of $428.8 \mathrm{~km}$ is suggested by Schaer (1999) as the optimum value and used in this research.

According to (12), the biggest change occurs for low elevations. For example, a change in elevation angle from $5^{\circ}$ to $15^{\circ}$ results in a change of the mapping function from 2.79-2.35. As a result of such changes in elevation angle, the residual ionospheric error may change by up to $0.34 \mathrm{~mm} / \mathrm{km}$ :

$0.78 \mathrm{~mm} / \mathrm{km} \cdot(2.79-2.35) \approx 0.34 \mathrm{~mm} / \mathrm{km}$

This variation reaches approximately $1 \mathrm{~cm}$ with a baseline of $30 \mathrm{~km}$. It is significantly smaller than the level of combined multipath errors in L1 and L2. Therefore, the residual ionospheric error can be considered as constant over such baselines and elevation changes. The carrierphase multipath errors tend to have sinusoidal patterns with zero mean for typical situations in which the reflected signal is much weaker than the direct signal (Ray and Cannon 1999). Hence, the constant terms in the measure-ments obtained from the second step can be removed by subtracting the mean of the measurements over a predetermined time-window. The IFM is obtained as:

$$
\begin{aligned}
\mathrm{IFM} & =\Delta m_{\mathrm{L} 1}^{k}-\Delta m_{\mathrm{L} 2}^{k} \\
& \approx \Delta \Phi_{\mathrm{L} 1}^{k}-\Delta \Phi_{\mathrm{L} 2}^{k}-\frac{1}{n} \sum_{t=1}^{n}\left(\Delta \Phi_{\mathrm{L} 1}^{t}-\Delta \Phi_{\mathrm{L} 2}^{t}\right)
\end{aligned}
$$

where $n$ is the number of epochs chosen in the computation of the mean. The optimum number of $n$ depends on the multipath frequency and the residual ionospheric error in the IFM observable. For short baselines, where residual ionospheric errors are negligible, the IFM accuracy increases with $n$. In general, for all baselines the optimal $n$ corresponds to one complete IFM cycle. The cycle-length relates to the reflector distance from the antenna. In the real data used in this experiment, cycle-lengths of $800-1,000 \mathrm{~s}$ were measured (Fig. 4).

Since the IFM observable for the in-between-receivers mode indicates the level of multipath for a given individual satellite, measurements with high multipath levels can be identified and discarded in the computation of the position solution. This method allows maximizing the use of carrier-phase measurements without a priori applying any elevation mask. The approach can also be used to choose the reference satellite with the lowest multipath level for RTK applications.

Between-satellites mode

The IFM observable between-satellites is derived using a similar approach for two satellites in close proximity, having similar elevation and azimuth angles. Signals from such satellites have correlated ionospheric errors, which are mitigated by the previously described process. Satellites in the same constellation are separated from each other by a long distance. Hence, the differencing process between the satellites cannot mitigate ionospheric errors. On the other hand, satellites from different constellations may be found in close proximity. Although different constellations use 
different time frames, the IFM generation is expected to be unaffected because the satellite clocks are stable over a long time and inter-constellation time differences can therefore be considered constant. As discussed previously, these constant biases are removed in the process of the IFM generation. The IFM observable in this mode is in particular useful to detect carrier-multipath errors in positioning techniques using only one receiver such as in precise point positioning (PPP).

\section{Double-differenced mode}

Double-difference carrier-phase measurements are obtained by differencing between-receiver measurements across satellites. The IFM observable in double-differenced mode is obtained for double-difference carrier-phase measurements in the same way as for the between-receivers mode. Receiver hardware delays are removed by double differencing. Therefore, there is no differential hardware delay (IFB). The double-differenced mode of IFM is very useful for multipath mitigation in linear combinations, discussed in the following sections.

\section{WL multipath mitigation using the IFM observable}

The double-differenced mode of the IFM observable theoretically can be used to estimate multipath errors in any linear combination that can be written as a difference between observations at two different frequencies. In order to use the IFM observable for multipath mitigation, Eq. (4) is rewritten based on the $\beta$ coefficient only. If $x$ is the difference between the two coefficients $\alpha$ and $\beta$ in (4) and $m_{c}$ is the carrier-multipath error in the combined measurement, then:

$$
\begin{aligned}
x= & \alpha-\beta \\
m_{c} & =(\beta+x) m_{\mathrm{L} 1}-\beta m_{\mathrm{L} 2} \\
& =\beta\left(m_{\mathrm{L} 1}-m_{\mathrm{L} 2}\right)+x m_{\mathrm{L} 1} \\
& =\beta \cdot I F M+x m_{\mathrm{L} 1}
\end{aligned}
$$

The IFM observable can be estimated using the process explained above, where $\beta$ is known. By correcting the measurement in the linear combination using the known part of (16), the carrier-multipath error $m_{c}$ is replaced by $x m_{\mathrm{L} 1}$ that corresponds to the carrier-multipath error in the L1 frequency scaled by the difference between the $\alpha$ and $\beta$ values. This approach is used here for carrier-multipath mitigation in the WL observables.

In the WL observable, the difference between parameters $\alpha$ and $\beta$ is $1(x=1)$. Therefore, by correcting the WL observable using the known part of (16), the carrier-multipath error is reduced to the level of the L1 carrier- multipath error. This means that the maximum values of carrier-multipath errors decrease from 42 to $4.6 \mathrm{~cm}$. The corrected WL is obtained as follows:

$$
\widehat{\Phi}_{1}=\Phi_{\mathrm{wl}}-\beta \cdot \mathrm{IFM}
$$

where $\Phi_{\mathrm{wl}}$ is the WL observable in units of length.

The correction based on (17) equally mitigates Gaussian noise, because it exhibits the same zero-mean characteristics as high-frequency carrier-multipath errors. In real-time applications, if the amount of historical data is smaller than one IFM cycle, it is possible to apply incorrect multipath corrections to the measurements. This can be avoided using two filters simultaneously before fixing the ambiguities, one with the corrected WL and the other without the cor-rection. The first correct set of ambiguities is accepted after passing a testing technique such as the ratio test (Feng et al. 2012).

\section{Data sets and testing cases}

Two data sets each of $1 \mathrm{~h}$ duration were collected using two Leica GS15 receivers in May 2012 at the Imperial College London Silwood Park campus (Silwood 1 and Silwood 2). The data were collected in a relatively open area with no vertical reflective surface in the vicinity of the antenna. The main source of reflection was the ground covered with wet grass. On the west side of the baseline, there was a sloping terrain below antenna level (Fig. 3). The sloping terrain may create forward scatter reflection for low elevation satellites with an azimuth of approxi-mately $270^{\circ}$. Forward scatter occurs when the signal is reflected from a horizontal surface below antenna level. Additionally, data were obtained from FARB, a surveyed permanent station.

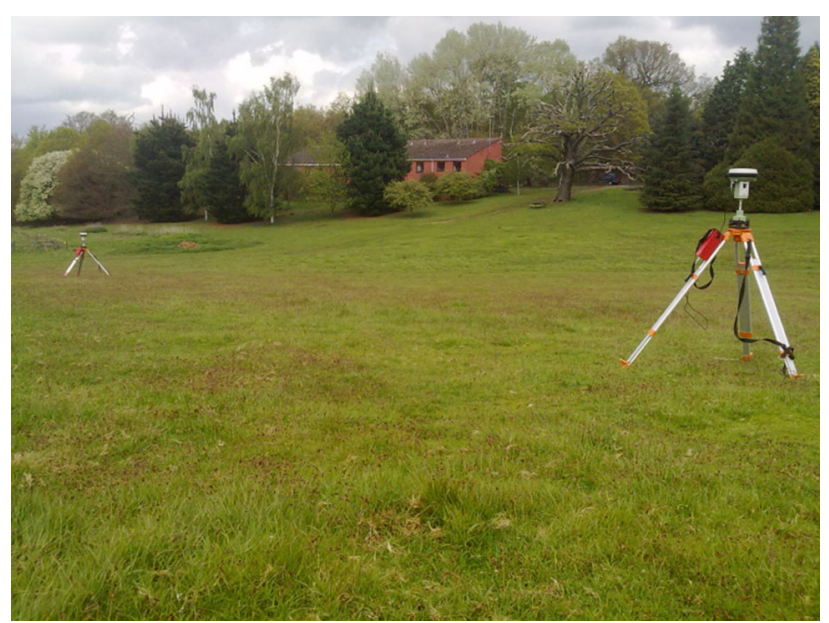

Fig. 3 Receiver settings for Silwood 1 and Silwood 2 data sets collections 
Three test cases were defined as follows:

- Case 1: Differencing between Silwood 1 and Silwood 2 with an approximate baseline of $20 \mathrm{~m}$.

- Case 2: Differencing between Silwood 1 and FARB, with an approximate baseline of $17 \mathrm{~km}$.

- Case 3: Differencing between Silwood 2 and FARB.

The IFM observable data for the between-receivers single-differenced modes were generated using raw data. To plot the IFM, the constant terms in (9) were removed by subtracting the mean over a period of $1 \mathrm{~h}$. Discontinuities larger than $1 \mathrm{~cm}$ in the IFM observable were assumed to be the result of cycle slips and were accordingly corrected in the computation of the mean. The IFM observable for the between-receivers mode for cases 1, 2 and 3 are illustrated in Fig. 4 top, middle and bottom panels, respectively. In
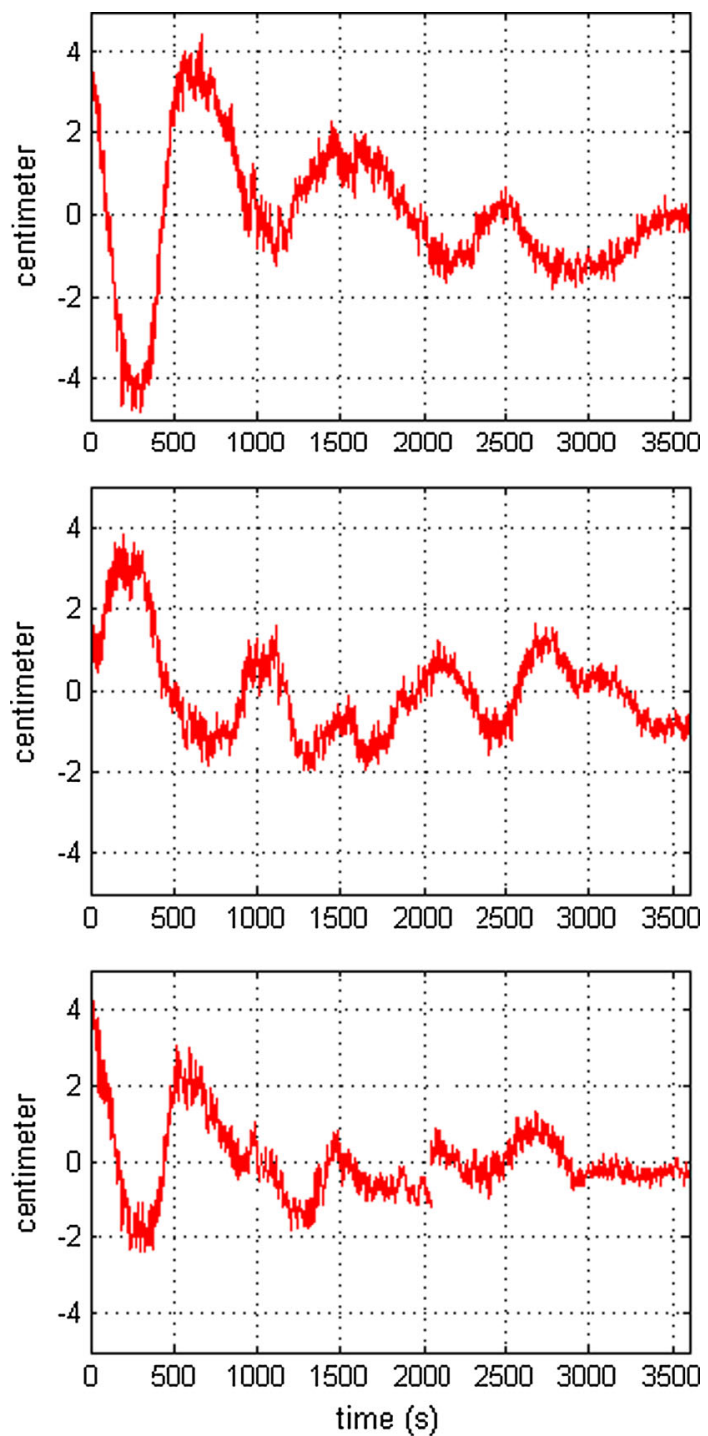

Fig. 4 Example of IFM observed $(p r n=9)$ in between-receivers mode for test case 1 (top), test case 2 (middle) and test case 3 (bottom) the plotted IFM interval, the elevation angle increased approximately linearly from $15^{\circ}$ to $40^{\circ}$. The IFM observables illustrated in the figures exhibit a sinusoidal pattern with zero mean, in line with expectations, as previously described.

To determine the level of carrier-multipath error for each individual satellite using the between-receiver mode of the IFM observable, observation over a sufficient time frame is required. In unknown operational environments, where the reflector distance from the antenna is not known, the number $n$ in (14) is progressively increased until a complete cycle in the IFM observable is reached.

Similar to the IFM in the between-receiver mode, the double-differenced modes were generated using the raw data. The IFM observable for the double-differenced mode of cases 1, 2 and 3 are illustrated in Fig. 5 top, middle and bottom panels, respectively. In the plotted IFM interval, the elevation angle increased approximately linearly from $15^{\circ}$ to $40^{\circ}$. The results in Figs. 4 and 5 show that the observables are similar for the between-receiver and double-differenced modes. This is expected because in the doubledifferenced mode, the satellite with the highest elevation angle was chosen as the reference satellite, which is typically less prone to multipath errors. Hence, the IFM observable in double-differenced mode was dominated by multipath in the secondary satellite.

\section{Testing the mitigation technique}

The reference positions for the Silwood 1 and Silwood 2 receivers were obtained in static mode using the GrafNav software, resolving ambiguities in RTK mode with FARB as the reference station. The position accuracies obtained by Grafnav software are $1.4 \mathrm{~cm}$ spherical error probable (SEP, as defined in (Leick 2004) and $4.8 \mathrm{~cm}$ SEP for Sil-wood 1 and Silwood 2, respectively. In each case, one station was used as the reference and the other as a rover and solutions were calculated epoch by epoch. The IFM observable in double-differenced mode was used to miti-gate the carriermultipath error in the WL observable. The iNsight project (www.insight-gnss.org) POINT software was used to test the method. In this software, ambiguity fixing is achieved using the Least-squares AMBiguity Decorrelation Adjustment (LAMBDA) method (Teunissen 1995).

The three test cases were conducted using an extended Kalman filter (EKF), commonly used in nonlinear stochastic systems for state estimation (Kaplan and Hegarty 2005). In the position calculation, the mean in (14) was calculated over a period of 1,000 s. Correcting the WL multipath based on (17) changes the correlation between the WL and L1 carrier-phase measurements. In order to 

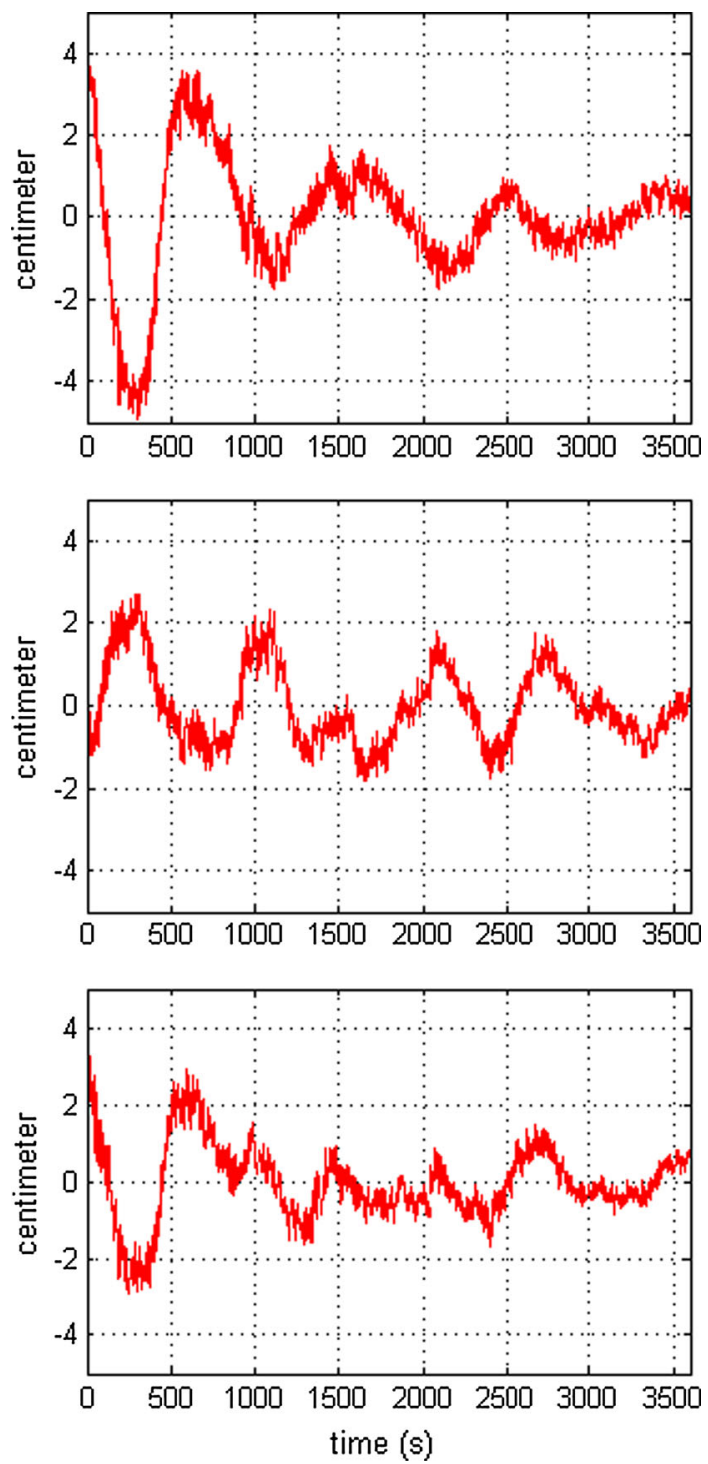

Fig. 5 Example of IFM observed (reference prn $=26$, secondary prn $=9)$ in double-differenced mode for test case 1(top), test case 2 (middle) and test case 3 (bottom)

analyze the impact of multipath correction in the WL and minimize the impacts of correlations, the WL is used as the only carrier-phase measurement in the first experiment. In the second experiment, the carrier-phase measurement in L2 is added to the observation vector in order to increase position accuracy. From the IFM analysis, relatively long initialization times are observed for some test cases. This can be attributed to the relatively high levels of carrierphase multipath in one of the secondary satellites $($ prn $=9)$ at the initial time.

\section{Experiment 1}

In this experiment, $\mathrm{L} 1$ code-phase measurements and WL carrier-phase observables were used. 3D position errors
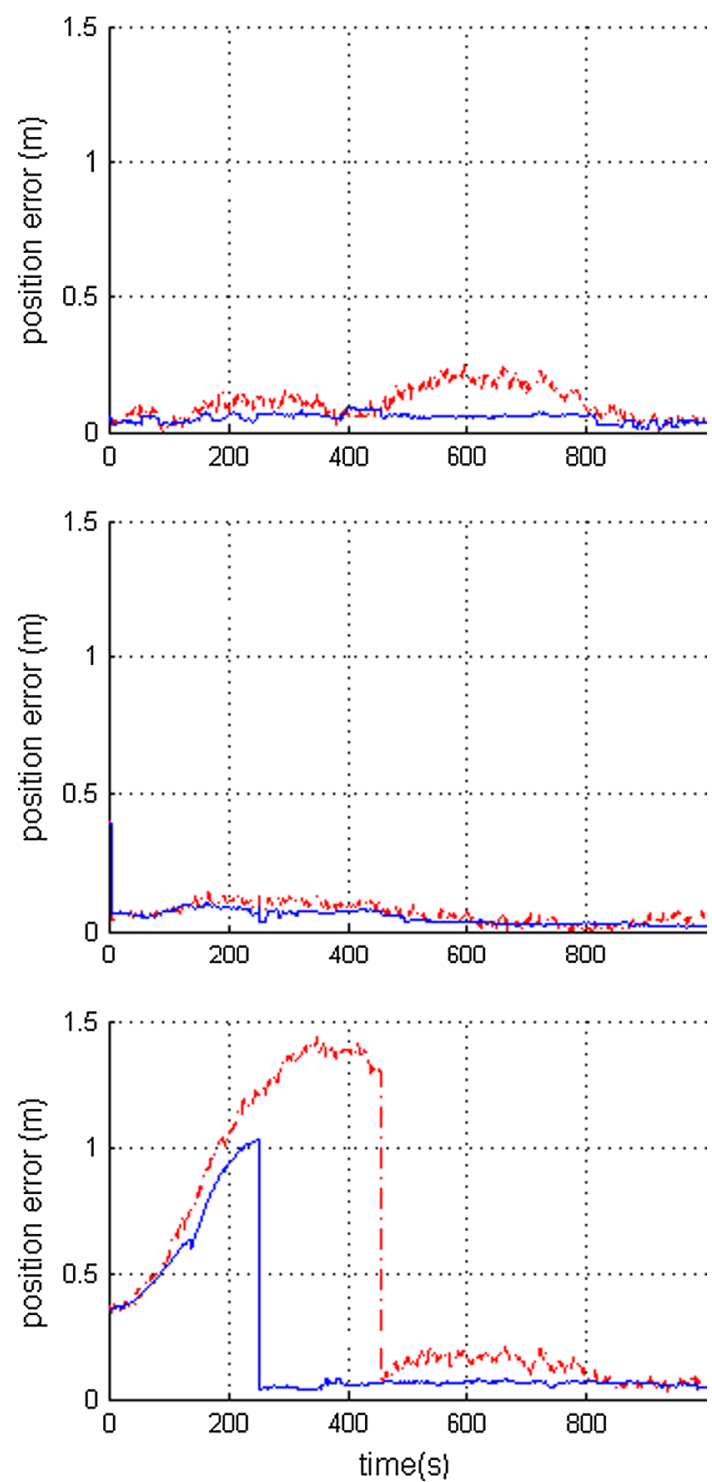

Fig. 6 3D position errors: no correction ( $r e d$ ) and multipath modeled (blue) for case 1 (top), case 2 (middle) and case 3 (bottom)

Table 1 Improvement in ambiguity fixing time for test cases 1, 2 and

\begin{tabular}{lllllll}
\hline $\begin{array}{l}\text { Test } \\
\text { case }\end{array}$ & $\begin{array}{l}\text { Baseline } \\
(\mathrm{km})\end{array}$ & \multicolumn{2}{l}{ Ambiguity fixing } & & \multicolumn{2}{l}{ Improvement } \\
\cline { 3 - 4 } \cline { 6 - 7 } & & $\begin{array}{l}\text { Before } \\
\text { correction }(\mathrm{s})\end{array}$ & $\begin{array}{l}\text { After } \\
\text { correction }(\mathrm{s})\end{array}$ & & $(\mathrm{s})$ & $(\%)$ \\
\hline 1 & 0.020 & 47 & 25 & & 22 & 47 \\
2 & 17 & 7 & 6 & & 1 & 14 \\
3 & 17 & 455 & 252 & & 203 & 45 \\
\hline
\end{tabular}

before and after carrier-phase multipath correction are illustrated in Fig. 6 top, middle and bottom panels for test cases 1,2 and 3 , respectively. The ambiguity resolution times and the improvements following multipath correction are summarized in Table 1. 

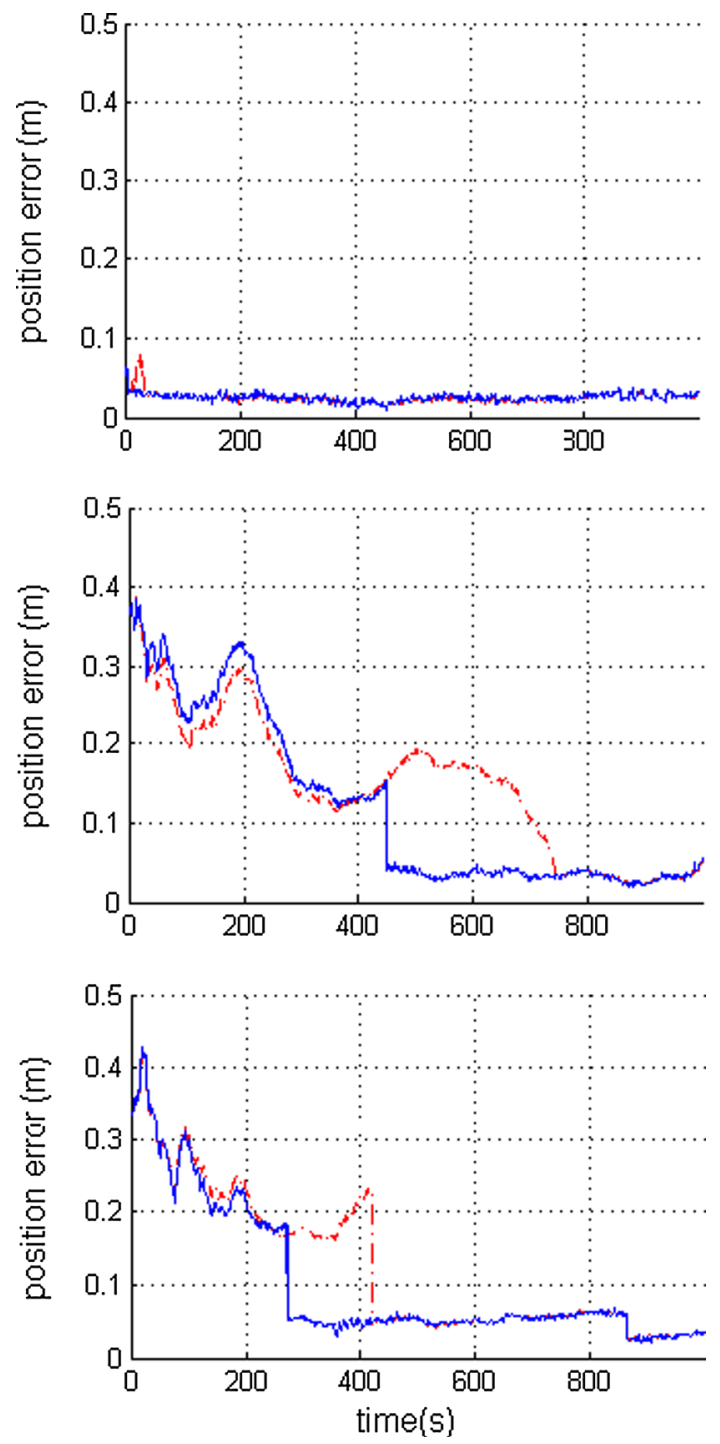

Fig. 7 3D position errors: no correction (red) and multipath modeled (blue) for case 1 (top), case 2 (middle) and case 3 (bottom)

The above results show that using the proposed method, the ambiguity fixing time has been reduced in all three cases. In case 3 , the ambiguities were fixed at a point where the position error was larger than at prior epochs. This may occur when biases are absorbed by the ambiguities at an early stage in the ambiguity resolution process. Case $2 \mathrm{had}$ a consider-able smaller fixing time than case 3 prior to and after mul-tipath correction. This difference appears to be associated with the difference in the multipath level between the two cases especially during the early epochs (Fig. 5 middle and bottom panels). Future research will further investigate the correlation between the WL and the code measurement to better understand this difference. Among all three cases, multipath errors were largest for case 1. Compare top panel with middle and bottom panels in Fig. 5. At the same time, due to the shorter baseline, the estimated IFM was most
Table 2 Improvement in ambiguity fixing time for test cases 1, 2 and 3

\begin{tabular}{lllllll}
\hline $\begin{array}{l}\text { Test } \\
\text { case }\end{array}$ & $\begin{array}{l}\text { Baseline } \\
(\mathrm{km})\end{array}$ & \multicolumn{2}{l}{ Ambiguity fixing } & & \multicolumn{2}{l}{ Improvement } \\
\cline { 3 - 4 } \cline { 6 - 7 } & & $\begin{array}{l}\text { Before } \\
\text { correction }(\mathrm{s})\end{array}$ & $\begin{array}{l}\text { After } \\
\text { correction }(\mathrm{s})\end{array}$ & & $(\%)$ \\
\hline 1 & 0.020 & 34 & 6 & 28 & 82 \\
2 & 17 & 744 & 452 & & 292 & 39 \\
3 & 17 & 424 & 273 & & 151 & 36 \\
\hline
\end{tabular}

accurate. Therefore, the improvement achieved in case 1 was best. The small discontinuity in the position error around 800 $\mathrm{s}$ in case one is due to a change in the number of satel-lites. Using only WL carrier-phase observables resulted in a noisy position calculation. The effect of noise and multipath errors was decreased significantly in the achieved position accuracy following multipath correction, as expected.

\section{Experiment 2}

In this experiment, code measurements in L1, carrier-phase measurements in L2 and WL carrier-phase observables were used. After applying corrections to the WL based on (17), the remaining WL multipath errors are correlated with the L1 carrier-phase multipath errors, which are considered to be noise by the Kalman filter. This noise correlation results in a non-optimal estimator of the Kal-man filter (Ma et al. 2010). It is therefore preferable to use L2 carrier-phase measurements instead of L1.

3D position errors before and after carrier-phase multipath correction are shown in Fig. 7 top, middle and bottom panels for test cases 1,2 and 3, respectively. The ambiguity resolution times and the improvements following multipath correction are summarized in Table 2.

The above results show that in this experiment, applying the multipath correction technique resulted in faster ambiguity fixing. However, results in Fig. 7 middle panel show that correcting multipath errors in the WL may also increase position errors. The correction of WL multipath errors changes the correlation between the L2 and WL carrier-phase measurements, potentially affecting the position error. In the bottom panel, there is a small dis-continuity in the position error after $800 \mathrm{~s}$, which is due to a change in the number of satellites.

\section{Conclusions and future work}

Carrier multipath is a significant error for high-accuracy positioning. State-of-the-art methods are unable to reliably model and mitigate these errors. The authors introduced a new method to detect and mitigate carrier-multipath error in linear combinations. 
The method was applied to mitigate carrier-phase multipath errors in the WL observable. The method reduces multipath errors in the WL observable to the level of L1 carrier-phase multipath, thereby significantly reducing the impact of multipath on the ambiguity resolution and posi-tion error.

To test the proposed method, two experiments were carried out using three test cases. The initial results show that the time required to obtain an initial ambiguity reso-lution was significantly reduced in both experiments. These results must be confirmed with more extensive testing in the future. To mitigate issues associated with the correla-tions between the carrier-phase measurements, it is best to exclusively use the multipath corrected WL before fixing the ambiguities. The L1 or L2 carrier-phase measurements should then be added to improve position accuracy. In the results described above, a fixed period $n$ of 1,000 was used to calculate the ambiguities and residual ionospheric errors in extracting the IFM and it was assumed that changes in the residual ionospheric errors are less than $1 \mathrm{~cm}$ over this period. This assumption is valid under nominal ionospheric conditions. Ionospheric conditions with high variations over time will limit the correction technique. Future work will develop an algorithm to determine a complete IFM cycle and remove the mean over this cycle.

\section{References}

Brenneman M, Morton Y, Yang C, van Grass F (2007) Mitigation of GPS multipath using polarization and spatial diversities. In: Proceedings of ION-GNSS-2007, Institute of Navigation, Fort Worth, TX, September 2007, pp 1221-1229

Christie J, Pervan B, Enge P, Powell J, Parkinson B, Ko P (1998) Analytical and experimental observations of ionospheric and tropospheric decorrelation effects for differential satellite navigation during precision approach. In: Proceedings of ION GPS, 1998. Institute of Navigation, pp 739-748

Feng S, Ochieng W, Samson J, Tossaint M, Hernandez-Pajares M, Juan JM, Sanz J, Aragón-Àngel À, Ramos-Bosch P, Jofre M (2012) Integrity monitoring for carrier phase ambiguities. J Navig 65(1):41-58

Gao Y, Heroux P, Kouba J (1994) Estimation of GPS receiver and satellite L1/L2 signal delay biases using data from CACS. In: Proceedings of KIS-94, Banff, Canada, August 30-September 2, pp 109-117

Jokinen A, Feng S, Schuster W, Ochieng W, Hide C, Moore T, Hill C, Milner C (2012) Improving fixed-ambiguity precise point positioning (PPP) convergence time and accuracy by using GLONASS. In: Proceedings of ION GNSS 2012, Institute of Navigation, Nashville, TN, September 2012, pp 3708-3727

Joosten P, Irsigler M (2003) GNSS ambiguity resolution in the presence of multipath. In: Proceedings of European navigation conference, Graz, Austria, p 15

Kaplan ED, Hegarty CJ (2005) Understanding GPS: principles and applications. Artech house, London

Komjathy A, Langley R (1996) An assessment of predicted and measured ionospheric total electron content using a regional
GPS network. In: Proceedings of the national technical meeting of the Institute of Navigation, pp 615-624

Lau L, Cross P (2007a) Development and testing of a new ray-tracing approach to GNSS carrier-phase multipath modelling. J Geod 81(11):713-732. doi:10.1007/s00190-007-0139-z

Lau L, Cross P (2007b) Investigations into phase multipath mitigation techniques for high precision positioning in difficult environments. J Navig 60(03):457-482. doi:10.1017/S0373463307004341

Leick A (2004) GPS satellite surveying, 3rd edn. Wiley, Canada

Ma L, Wang H, Chen J (2010) Analysis of Kalman filter with correlated noises under different dependence. J Inf Comput Sci 7(5):1147-1154

Manandhar D, Shibasaki R, Normark PL (2004) GPS signal analysis using LHCP/RHCP antenna and software GPS receiver. In: Proceedings of ION-GNSS-2004, Long Beach, CA, pp 2489-2498

Mannucci A, Wilson B, Yuan D, Ho C, Lindqwister U, Runge T (1998) A global mapping technique for GPS-derived ionospheric total electron content measurements. Radio Sci 33(3):565-582

Moradi R, Schuster W, Feng S, Ochieng W (2013) Reducing GPS wide lane ambiguity resolution time: a novel carrier phase multipath mitigation technique. In: Proceedings of ION-ITM2013, Institute of Navigation, San Diego, CA, January 29-27, pp 343-350

Ray J, Cannon M (1999) Characterization of GPS carrier phase multipath. In: Proceedings of ION-NTM-1999, San Diego, CA, January 25-27, pp 343-352

Reichert A, Axelrad P (1999) GPS carrier phase multipath reduction using SNR measurements to characterize an effective reflector. In: Proceedings of ION-GPS-1999, Nashville, TN, September 14-17, pp 1951-1960

Schaer S (1999) Mapping and predicting the Earth's ionosphere using the global positioning system. PhD Dissertation, University of Bern

Schuster W, Ochieng W (2011) Novel integrity concept for CAT III precision approaches and taxiing: extended GBAS (E-GBAS). J Navig 64(4):695-710

Teunissen P (1995) The least-squares ambiguity decorrelation adjustment: a method for fast GPS integer ambiguity estimation. J Geod 70(1):65-82

Van Dierendonck AJ, Fenton P, Ford T (1992) Theory and performance of a narrow correlator spacing in a GPS receiver. Navig J ION 39(3):265-283

Wanninger L, May M (2001) Carrier phase multipath calibration of GPS reference stations. Navig J ION 48(2):113-124 